\title{
Hospitais de Cidades Pequenas: inserção e pactuação no Sistema Único de Saúde (SUS)
}

\author{
Small Towns Hospitals: insertion and agreement in the Brazilian Unified \\ Health System
}

Francisco Eugênio Alvez de Souza ${ }^{1}$ (D), Elisabete de Fátima Polo de Almeida Nunes ${ }^{1}$ (D), Brigida Gimenez Carvalho ${ }^{1}$ (D), Fernanda de Freitas Mendonça ${ }^{1}$ (D)

\begin{abstract}
RESUMO
Importância do problema: É indiscutível a importância do hospital na organização no Sistema Único de Saúde (SUS). Os hospitais com 50 leitos ou menos, também chamados de hospitais de pequeno porte (HPP) são essenciais para a construção das redes de atenção à saúde (RAS) considerando sua capilaridade no sistema. Objetivo: Descrever a caracterização dos hospitais de pequeno porte e sua adequação à política vigente. Método: Trata-se de um estudo transversal, descritivo, realizado a partir de dados secundários obtidos de bases de dados nacionais: Cadastro Nacional de Estabelecimentos de Saúde (CNES-MS), Sistema de Informações Hospitalares (SIH/SUS) e o sistema de registro de dados hospitalares da Regional de Saúde. O estudo foi realizado em uma região de saúde localizada no norte do Paraná, Brasil. Resultados: Neste estudo, 100\% dos hospitais em cidades pequenas da 17a Regional de Saúde do Paraná são de pequeno porte. A oferta de leitos atende à estabelecida pela portaria do Ministério da Saúde n 1.101/2002. Observou-se a extrema ociosidade da capacidade instalada, centros cirúrgicos inativos e baixíssima ocupação dos leitos hospitalares. Conclusão: Conclui-se que a forma de pactuação/contratualização isolada de cada hospital não surtiu os efeitos desejados pela Política Nacional de HPP (PNHPP). Sugere-se que uma nova vinculação ocorra de forma regionalizada e que se criem um sistema unificado de gestão.
\end{abstract}

Palavras-chave: Administração Pública; Administração Hospitalar; Tamanho das Instituições de Saúde; Cidades Pequenas.

\begin{abstract}
Importance of the problem: The importance of the hospital in the organization of the Brazilian Unified Health System (UHS) is indisputable. Hospitals with 50 beds or fewer, also called small hospitals are essential for building health care networks considering their capillarity in the system. Objective: Describing the characterization of small hospitals and their adequacy to current policy and confirm the results presented so far in the literature. Method: This is a cross-sectional, descriptive study based on secondary data obtained from the national databases: National Registry of Health Establishments, Hospital Information System, and registry of data of the Regional of Health. The study was conducted in a health region located in northern Paraná, Brazil. Results: In this study, more than $90 \%$ of the hospitals in towns of the 17th Regional of Health of Paraná are small. The supply of beds meets the guidelines of the Ministry of Health n. 1,101/2002. It was observed the extreme idleness of facilities, inactive surgical centers, and low occupation of hospital beds. Conclusion: It is concluded that the form of agreement isolated from each hospital did not have the desired effects by national small hospital policy. It is suggested that a new connection occurs regionally and that a unified management system might be created.
\end{abstract}

Keywords: Public Administration; Hospital Administration; Health Facility Size; Cities.

1. Universidade Estadual de Londrina (UEL), Londrina (PR), Brasil.

$\bowtie$ Fernanda de Freitas Mendonça. Departamento de Saúde Coletiva. Centro de Ciências da Saúde. Universidade Estadual de Londrina. Avenida Robert Koch, 60 - Vila Operária. CEP: 86039-440. Londrina (PR), Brasil.

fernanda0683@gmail.com | Recebido em: 23/03/2020 | Aprovado em: 03/07/2020 


\section{INTRODUÇÃO}

É indiscutível a importância do hospital na organização no Sistema Único de Saúde (SUS), seja pelo tipo de serviços ofertados, seja pelo volume considerável de recursos destinado a esse nível de atenção. O setor hospitalar, além de realizar internações, é também responsável por um quarto das consultas ambulatoriais e por quase $80 \%$ dos atendimentos de urgência e emergência. Estima-se que sua participação nos gastos do setor saúde seja de aproximadamente $67,0 \%{ }^{1}$.

Os hospitais podem ser classificados de acordo com o número de leitos em: Pequeno porte: com capacidade de operação de até 50 leitos; Médio porte: de 51 a 150 leitos; Grande porte: de 151 a 500 leitos e acima de 500 leitos considera-se hospital de capacidade extra².

Em 2017, existiam no Brasil 5819 hospitais registrados no CNES e, destes, $60 \%$ tinham até 50 leitos e apenas $16,2 \%$ possuíam 100 leitos ou mais $^{3}$. Os hospitais com menos de 50 leitos, também chamados de HPP se distribuem de maneira heterogênea nas regiões brasileiras, sendo que na Região Centro-Oeste correspondiam a $78 \%$ dos hospitais, enquanto na Região Sudeste esta proporção era de $48 \%^{3}$. Frequentes no país, os HPP estão distribuídos, majoritariamente, em municípios interioranos e com dimensões demográficas reduzidas, os pequenos municípios ${ }^{4}$, garantindo acesso à saúde para a população residente nestas localidades.

No entanto, se por um lado garantem algum tipo de acesso, por outro oferecem uma atenção hospitalar pouco eficiente e resolutiva ${ }^{5}$. Segundo a Diretora do Departamento de Atenção Hospitalar e de Urgência do Ministério da Saúde, quanto menor o porte do hospital, menores são as suas taxas de ocupação, chegando ao patamar de $22 \%$ nos hospitais com menos de 50 leitos $^{6}$. Além disso, esses estabelecimentos possuem, em geral, baixa resolutividade, má relação custo-efetividade e, via de regra, não se caracterizam como um ponto de atenção em rede, ou seja, não têm claro qual sua missão nas RAS ${ }^{5,7}$.

Os HPP, apesar de terem produzido (no ano de 2005$), 21 \%$ das AIH pagas, faturaram apenas $12 \%$ do valor total pago pelo SUS a título de internações, o que se deve à baixa complexidade de suas internações $e$, consequentemente ao baixo valor da sua produção ${ }^{4}$.
Especialistas em Economia da Saúde ${ }^{8}$ corroboram essa análise e acrescentam que, dessa forma, a saúde financeira dos HPP é sempre prejudicada, visto que apresentam receitas SUS menores que seus custos. Além disso, as atividades e procedimentos de baixa complexidade são mal remuneradas nesses estabelecimentos, que recebem somente cerca de $25 \%$ do custo real dos procedimentos realizados.

No ano de 2004, com intuito de estimular a inserção dos HPP na rede hierarquizada de atenção à saúde, foi instituída, por meio da Portaria no 1044/GM, a PNHPP. Por esta política, hospitais com menos de 30 leitos foram estimulados a aderir voluntariamente a um processo de contratualização (com as Secretarias Estaduais de Saúde), com redefinição de seu papel na rede de serviços, estabelecimento de metas quantitativas e qualitativas, e de um orçamento global, em substituição ao pagamento por internações ${ }^{9}$.

Após a implantação da PNHPP, um estudo ${ }^{4}$ (p.927) realizou uma avaliação do desempenho dos HPP, no período de 2004 a 2006. Como resultado, não foi observado grande mudança no perfil de produção desses hospitais. Concluem que, "se analisados a estrutura e o perfil de sua produção, os hospitais de pequeno porte, no Brasil, não aderiram suficientemente à indução da redefinição do seu perfil". Uma das hipóteses apontada por esse resultado, teria sido o breve espaço de tempo após a contratualização desses hospitais e a coleta dos dados da referida pesquisa.

Considerando o papel estratégico que os HPP exercem nas RAS, sobretudo, pela sua capilaridade no sistema, além da pouca realização de estudos acerca deste tema e decorridos 15 anos da edição da PNHPP, propõe-se a realização de um novo estudo, em uma região composta por 14 HPP, todos localizados em pequenas cidades. $\mathrm{O}$ objetivo foi descrever a caracterização dos hospitais de pequeno porte e sua adequação à política vigente, por meio da análise da capacidade instalada, e de alguns indicadores de desempenho desses hospitais.

\section{METODOLOGIA}

Trata-se de um estudo transversal, descritivo, sobre os hospitais localizados em cidades 
pequenas, com população de até 20.000 habitantes, pertencentes a uma região de saúde, localizada no norte do Estado do Paraná. Este estudo é parte de uma tese de doutorado intitulada Hospitais em "Municípios de Pequeno Porte: sua inserção no SUS". Este recorte da pesquisa foi realizado a partir de dados secundários, e foram analisados os seguintes aspectos: identificação dos municípios e hospitais, população, natureza jurídica das instituições, infraestrutura dos hospitais e indicadores hospitalares (taxa de leitos por habitantes, valor médio da internação, média mensal de internação, média mensal de internação/leito, proporção de internações eletivas e de urgência).

Os dados foram obtidos de bases de dados nacionais tais como: CNES-MS e SIH/SUS acessados por meio dos sítios eletrônicos http://cnes. datasus.gov.br/ e http://sihd.datasus.gov.br, respectivamente, e se referem ao ano de 2018. Além disso, também foram utilizados registro de dados hospitalares da Regional de Saúde, obtidos diretamente com esse serviço. Tais dados foram levantados no período de maio a junho de 2019. Foi realizada análise descritiva (frequência absoluta e relativa), utilizando-se o programa Microsoft Excel.
O projeto deste estudo foi aprovado pelo Comitê de Ética em Pesquisa da instituição a que os pesquisadores estão vinculados, com parecer número 1.440 .160 e CAAE:53211215200005231.

\section{RESULTADOS}

A 17a Regional de Saúde, da Secretaria de Estado da Saúde (SESA) - PR é constituída por 21 municípios, destes 17 são MPP, e 14 deles têm hospitais com menos de 50 leitos. A Tabela 1 apresenta a relação dos hospitais nestes municípios, a natureza jurídica e a distribuição dos leitos.

Em relação à entidade mantenedora, 13 hospitais são públicos municipais $(92,8 \%)$ e apenas um é privado com fins lucrativos. Dos $13 \mathrm{HPP}$ públicos, oito estão contratualizados junto à Secretaria de Saúde do Estado, com vistas à implantação da PNHPP.

O total de leitos ofertados por estes hospitais é de 296, o que corresponde a 1,9 leitos por mil habitantes nesta região. Do total de leitos dos 14 hospitais, um pouco mais da metade, 154 leitos (52\%) são clínicos, 61 obstétricos $(20,6 \%), 63$ pediátricos $(21,2 \%)$ e 18 cirúrgicos (6\%). (Tabela 2).

\section{Tabela 1}

Distribuição dos Hospitais em municípios de pequeno porte da $17^{a}$ Regional de Saúde, população, entidade mantenedora e leitos por hospital, Paraná, 2018.

\begin{tabular}{lcccc}
\hline Município & População & Natureza Jurídica & Total de Leitos & Leitos/1.000 Hab \\
\hline Alvorada do Sul & 11.306 & Público/Municipal & 16 & 1,4 \\
Assai & 15.289 & Público/Municipal & 38 & 2,5 \\
Bela Vista do Paraíso & 15.395 & Público/Municipal & 19 & 1,2 \\
Centenário do Sul & 10.891 & Público/Municipal & 25 & 2,3 \\
Florestópolis & 10.646 & Público/Municipal & 22 & 2,1 \\
Jaguapitã & 13.494 & Público/Municipal & 29 & 2,1 \\
Jataizinho & 12.536 & Privado/Sociedade Emp. Ltda. & 19 & 1,5 \\
Lupionópolis & 4.894 & Público/Municipal & 16 & 3,3 \\
Miraselva & 1.816 & Público/Municipal & 10 & 5,5 \\
Porecatu & 13.084 & Público/Municipal & 31 & 2,4 \\
Prado Ferreira & 3.726 & Público/Municipal & 20 & 19 \\
Primeiro de Maio & 11.114 & Público/Municipal & 19 & 1,7 \\
Sertanópolis & 16.323 & Público/Municipal & 16 & 1,0 \\
Tamarana & 14.548 & Público/Municipal & 296 & 1,1 \\
\hline Total & $\mathbf{1 5 5 . 0 6 2}$ & & 1,9 & \\
\hline
\end{tabular}




\section{Tabela 2}

Distribuição do número de leitos hospitalares por clínica, nos municípios de pequeno porte da $17 a$ Regional de Saúde do Paraná, 2018.

\begin{tabular}{|c|c|c|c|c|c|c|c|c|c|}
\hline \multirow{2}{*}{ Municípios } & \multicolumn{2}{|c|}{ Cirúrgico } & \multicolumn{2}{|c|}{ Clínico } & \multicolumn{2}{|c|}{ Obstétrico } & \multicolumn{2}{|c|}{ Pediátrico } & \multirow{2}{*}{ Total } \\
\hline & $\mathbf{N}$ & $\%$ & $\mathbf{N}$ & $\%$ & $\mathbf{N}$ & $\%$ & $\mathbf{N}$ & $\%$ & \\
\hline Alvorada do Sul & - & & 10 & 62,5 & 3 & 18,8 & 3 & 18,8 & 16 \\
\hline Assai & 6 & 15,8 & 12 & 31,6 & 6 & 15,8 & 14 & 36,8 & 38 \\
\hline Bela Vista do Paraíso & 2 & 10,5 & 8 & 42,1 & 5 & 26,3 & 4 & 21,1 & 19 \\
\hline Centenário do Sul & - & & 12 & 48,0 & 7 & 28,0 & 6 & 24,0 & 25 \\
\hline Florestópolis & 1 & 4,5 & 11 & 50,0 & 6 & 27,3 & 4 & 18,2 & 22 \\
\hline Jaguapitã & 2 & 6,9 & 19 & 65,5 & 4 & 13,8 & 4 & 13,8 & 29 \\
\hline Jataizinho & 2 & 10,5 & 10 & 52,6 & 4 & 21,1 & 3 & 15,8 & 19 \\
\hline Lupionópolis & - & & 8 & 50,0 & 5 & 31,3 & 3 & 18,8 & 16 \\
\hline Miraselva & - & & 6 & 60,0 & 2 & 20,0 & 2 & 20,0 & 10 \\
\hline Porecatu & 4 & 12,9 & 15 & 48,4 & 6 & 19,4 & 6 & 19,4 & 31 \\
\hline Prado Ferreira & - & & 14 & 70,0 & 3 & 15,0 & 3 & 15,0 & 20 \\
\hline Primeiro de Maio & - & & 9 & 47,4 & 5 & 26,3 & 5 & 26,3 & 19 \\
\hline Sertanópolis & - & & 12 & 75,0 & 2 & 12,5 & 2 & 12,5 & 16 \\
\hline Tamarana & 1 & 6,3 & 8 & 50,0 & 3 & 18,8 & 4 & 25,0 & 16 \\
\hline Total & 18 & 6,0 & 154 & 52,0 & 61 & 20,6 & 63 & 21,2 & 296 \\
\hline
\end{tabular}

Essa maior proporção de leitos clínicos ocorre em 13 hospitais. Apenas o hospital com 38 leitos apresentava uma distribuição mais homogênea nas diferentes clínicas, sendo a oferta de leitos pediátricos até superior aos clínicos.

Estes hospitais possuíam cadastro de estruturas destinadas ao atendimento ambulatorial (consultórios, sala de curativo, sala de observação, dentre outras). Além dessa estrutura, todos os hospitais contavam com uma ou mais salas de cirurgia cadastradas, e a maioria possuía também sala de recuperação, parto e pré-parto.

A Tabela 3 apresenta a distribuição de internações por tempo de permanência e caráter de atendimento. O número total de internações hospitalares no período de janeiro a dezembro de 2018 foi de 3918. O hospital com o maior número de internações foi em Jataizinho, com 839 representando $21,4 \%$ do total e o menor foi em Miraselva com 28 internações no ano $(0,71 \%)$. O maior número de internações teve duração de dois a quatro dias $(72,4 \%)$, o que foi comum para todos os hospitais estudados. A quase totalidade das internações $(94,4 \%)$ ocorreu em caráter de urgência.

A Tabela 4 apresenta as internações por especialidade, por valor médio da internação, número médio mensal de internações por hospital e por leito.

Observa-se que as internações clínicas são as mais recorrentes das realizadas pelos hospitais $(83,3 \%)$, seguidas das obstétricas $(6,1 \%)$, pediátricas $(5,2 \%)$ e cirúrgicas $(0,9 \%)$.

Além disso, apenas três hospitais apresentaram internações cirúrgicas e vários realizaram internações pediátricas e obstétricas, embora não possuíssem médicos especialistas destas áreas cadastrados no CNES.

O número médio mensal de internações foi de 326,5 para o total de 296 leitos, alcançando a irrisória taxa de 1,1 internações mensais por leito, em média. A menor taxa foi de 0,2 e a maior 2,6 entre os hospitais públicos. O único hospital privado apresentou a média de 3,6 internações/leito por mês, destoante do perfil dos demais HPP estudados.

O valor médio das internações dos hospitais foi de $R \$ 428,70$ variando de $R \$ 232,52$ a $R \$ 597,40$ para as internações realizadas no hospital privado. Destaca-se que, apesar de apresentar o maior valor médio, esta instituição privada não apresentou internações cirúrgicas e tem cadastrados poucos equipamentos e serviços especializados nos registros do CNES, o que leva a supor a existência de distorções na emissão das AIH para esta instituição. 
Tabela 3

Distribuição das internações hospitalares por tempo de permanência e caráter de atendimento nos municípios de pequeno porte da 17a Regional de Saúde do Paraná, 2018.

\begin{tabular}{|c|c|c|c|c|c|c|c|c|c|c|c|}
\hline \multirow{2}{*}{ Município } & \multirow{2}{*}{$\begin{array}{c}0 \\
\text { dia }\end{array}$} & \multirow{2}{*}{$\begin{array}{c}1 \\
\text { dia }\end{array}$} & \multirow{2}{*}{$\begin{array}{l}2-4 \\
\text { dias }\end{array}$} & \multirow{2}{*}{$\begin{array}{l}5-7 \\
\text { dias }\end{array}$} & \multirow{2}{*}{$\begin{array}{l}\text { 8-14 } \\
\text { dias }\end{array}$} & \multirow{2}{*}{$\begin{array}{c}\text { 15-21 } \\
\text { dias }\end{array}$} & \multirow{2}{*}{$\begin{array}{c}22-28 \\
\text { dias }\end{array}$} & \multirow{2}{*}{$\begin{array}{c}29 \text { dias } \\
e+\end{array}$} & \multirow{2}{*}{\multicolumn{3}{|c|}{$\begin{array}{l}\text { Eletivo Urgência Total } \\
\text { (Internações Hospitalares }\end{array}$}} \\
\hline & & & & & & & & & & & \\
\hline Alvorada do Sul & - & 17 & 102 & 32 & 14 & 1 & - & - & 165 & 1 & 166 \\
\hline Assaí & 26 & 90 & 360 & 58 & 19 & - & - & - & - & 553 & 553 \\
\hline Bela V Paraíso & 20 & 68 & 423 & 69 & 20 & 1 & 1 & 1 & 53 & 550 & 603 \\
\hline Centenário Sul & - & - & 213 & 10 & 2 & 1 & - & - & - & 226 & 226 \\
\hline Florestópolis & - & 12 & 43 & 22 & 5 & - & - & - & - & 82 & 82 \\
\hline Jaguapitã & 7 & 45 & 190 & 68 & 16 & 3 & - & - & - & 329 & 329 \\
\hline Jataizinho & 2 & 8 & 795 & 27 & 5 & - & - & 2 & - & 839 & 839 \\
\hline Lupionópolis & - & 14 & 61 & 3 & - & - & - & - & - & 78 & 78 \\
\hline Miraselva & - & 6 & 12 & 9 & - & - & - & 1 & - & 28 & 28 \\
\hline Porecatu & 1 & 42 & 207 & 57 & 13 & 1 & - & 2 & - & 323 & 323 \\
\hline Prado Ferreira & - & - & 48 & 13 & - & 1 & - & - & - & 62 & 62 \\
\hline Primeiro Maio & 1 & 6 & 60 & 44 & 10 & 7 & - & - & - & 128 & 128 \\
\hline Sertanópolis & - & 3 & 147 & 50 & 21 & 1 & 2 & - & - & 224 & 224 \\
\hline Tamarana & 1 & 29 & 176 & 52 & 17 & 1 & 1 & - & - & 277 & 277 \\
\hline Total & 58 & 340 & 2837 & 514 & 142 & 17 & 4 & 6 & 218 & 3700 & 3918 \\
\hline
\end{tabular}

\section{Tabela 4}

Internações por especialidade, valor médio da internação, média mensal de internações e taxa de internação por leito nos MPP da 17a RS do Paraná, 2018.

\begin{tabular}{|c|c|c|c|c|c|c|c|c|c|}
\hline Município & Cir. & Obst. & Clínic. & Ped. & Total & $\begin{array}{l}\text { Valor total } \\
\text { internações }\end{array}$ & $\begin{array}{c}\text { Valor } \\
\text { médio de } \\
\text { internação }\end{array}$ & $\begin{array}{c}\text { Taxa de } \\
\text { internação } \\
\text { por mês }\end{array}$ & $\begin{array}{l}\text { Taxa internação } \\
\text { mensal por leito }\end{array}$ \\
\hline $\begin{array}{l}\text { Alvorada do } \\
\text { Sul }\end{array}$ & 0 & 0 & 166 & 0 & 166 & $\mathrm{R} \$ 45.948,60$ & $R \$ 345,48$ & 13,88 & 0,86 \\
\hline Assaí & 30 & 65 & 431 & 27 & 553 & $R \$ 142.674,52$ & $R \$ 363,97$ & 46,08 & 1,21 \\
\hline $\begin{array}{l}\text { Bela Vista do } \\
\text { Paraíso }\end{array}$ & 4 & 164 & 389 & 46 & 603 & $\mathrm{R} \$ 245.905,63$ & $R \$ 438,33$ & 50,25 & 2,64 \\
\hline $\begin{array}{l}\text { Centenário do } \\
\text { Sul }\end{array}$ & 0 & 2 & 216 & 8 & 226 & $R \$ 101.576,73$ & $\mathrm{R} \$ 389,18$ & 18,33 & 0,75 \\
\hline Florestópolis & 2 & 0 & 80 & 0 & 82 & $R \$ 39.086,77$ & $R \$ 402,96$ & 6,83 & 0,23 \\
\hline Jaguapitã & 0 & 1 & 287 & 41 & 329 & $R \$ 154.928,77$ & $R \$ 406,64$ & 27,41 & 0,94 \\
\hline Jataizinho & 0 & 1 & 838 & 0 & 839 & $R \$ 496.438,63$ & $R \$ 597,40$ & 69,91 & 3,67 \\
\hline Lupionópolis & 0 & 0 & 75 & 3 & 78 & $\mathrm{R} \$ 38.944,28$ & $\mathrm{R} \$ 301,89$ & 6,5 & 0,40 \\
\hline Miraselva & 0 & 0 & 26 & 2 & 28 & $\mathrm{R} \$ 6.510,63$ & $R \$ 232,52$ & 2,33 & 0,23 \\
\hline Porecatu & 0 & 0 & 133 & 21 & 323 & $\mathrm{R} \$ 50.401,72$ & $R \$ 327,28$ & 26,91 & 0,86 \\
\hline Prado Ferreira & 0 & 0 & 61 & 1 & 62 & $R \$ 17.348,30$ & $R \$ 228,27$ & 5,16 & 0,25 \\
\hline $\begin{array}{l}\text { Primeiro de } \\
\text { Maio }\end{array}$ & 0 & 0 & 128 & 0 & 128 & $R \$ 26.636,82$ & $R \$ 403,59$ & 10,66 & 0,56 \\
\hline Sertanópolis & 0 & 0 & 215 & 9 & 224 & $\mathrm{R} \$ 72.331,31$ & $R \$ 404,09$ & 18,66 & 1,16 \\
\hline Tamarana & 0 & 9 & 222 & 46 & 277 & $\mathrm{R} \$ 63.004,13$ & $R \$ 293,04$ & 23,08 & 1,44 \\
\hline Total & 36 & 242 & 3267 & 204 & 3918 & R\$ 1.501.736,84 & $R \$ 428,70$ & 326,5 & 1,10 \\
\hline
\end{tabular}




\section{DISCUSSÃO}

Os HPP representam hoje mais de $50 \%$ das instituições hospitalares do país e estão distribuídos de forma bastante capilarizada em termos geográficos, atuando de maneiras distintas e de acordo com sua localização ${ }^{5,6}$. Isso se verifica na região estudada, pois dentre os 17 municípios com menos de 20 mil habitantes da região, apenas três não possuem hospital em seu território.

Em relação à oferta de leitos hospitalares à população, constata-se que esta tem diminuído na maior parte do mundo desenvolvido. Os avanços tecnológicos, novas drogas, procedimentos e técnicas reduzem fortemente a permanência hospitalar permitindo melhor aproveitamento (giro) dos leitos existentes ${ }^{10}$. Estudos apontam a ocorrência de fusões e alianças estratégicas de hospitais em países da Europa, objetivando melhorar o desempenho, a competitividade e os serviços fornecidos aos usuários ${ }^{11,12}$.

Ao avaliar a oferta de leitos por esses hospitais na região, verifica-se que nos 14 municípios com HPP, o número de leitos existentes supera o recomendado. Chega-se a essa conclusão ao aplicar os parâmetros estabelecidos pela PNHPP, com intuito de redefinir a necessidade de leitos desses hospitais, baseados em critérios populacionais e parâmetros hospitalares ${ }^{13}$. Por esses parâmetros, o número de leitos ajustados para esses municípios seria de 120, o que é bem inferior aos 296 disponíveis nos 14 municípios.

Considera-se que um desenho de necessidade de leitos, fundamentado no perfil epidemiológico regional, que tenha clareza da missão de cada estabelecimento hospitalar nas RAS, e que promova a resolutividade dos leitos deve preceder às decisões de construção de hospitais ${ }^{6}$.

No entanto, essa recomendação certamente não foi adotada na região estudada porque a maioria desses hospitais existe de longa data, alguns deles eram hospitais privados e foram municipalizados à época da implantação do SUS e estudos aprofundados sobre a necessidade da manutenção desses hospitais na região não ocorreu. Outros fatores diferentes da questão da eficácia dos hospitais, enquanto instituições de saúde, foram levados em consideração para a decisão de manutenção dos mesmos em descumprimento às normatizações legais ${ }^{7}$.
Esses fatores podem ser entendidos como um conjunto de valores de cunho simbólico/cultural que, assim como os fatores objetivos que organizam as instituições, exerce tanta pressão para o funcionamento da sociedade como os demais. Essa ideia pode ser melhor apresentada compreendendo algumas teorias de Pierre Bourdieu, sociólogo francês da segunda metade do século $X X^{14}$.

O principal conceito da teoria formulada por Bourdieu é o de poder simbólico. Segundo o autor, a organização da sociedade é marcada pela subjetividade de sua configuração e construída em meio a uma série de valores que implicam numa diferenciação entre os indivíduos. Esses valores são múltiplos e nem sempre materiais ou visíveis, obedecem e são, ao mesmo tempo, determinantes da construção social. Parece assim, que o poder simbólico ocupa uma função central na organização da sociedade que, mesmo aceitando sua existência descontínua, mantém usando desses valores como forma de organização das ações dos indivíduos em sociedade ${ }^{14}$.

A proposta é pensar a questão da valorização dos hospitais dentro da lógica esboçada por Bourdieu e interrogar que tipo de capitais simbólicos e culturais os hospitais representam enquanto instituição social, a fim de perceber porque mesmo com questionamentos sobre a real eficácia destes para a questão de saúde, os municípios de pequeno porte ainda os mantêm.

É provável que este poder simbólico esteja alicerçado no modelo hospitalocêntrico, ainda hegemônico no SUS, o que contribui para reforçar os problemas do setor saúde. O modelo, centrado no hospital, predominantemente inserido no imaginário popular, está historicamente associado à forma como o Brasil, ao longo dos anos, enfrentou os problemas de saúde, buscando solucioná-los basicamente a partir da doença e predominantemente em instituições hospitalares ${ }^{6}$.

Além do mau desempenho, isto é, de sua baixa produtividade em termos do número de internações produzidas, questiona-se também sobre a resolutividade dessas internações, ou seja, se seriam de fato necessárias. Seria preciso repensar a realidade dos leitos, pois um hospital de 50 leitos precisaria atender a várias cidades de 20 mil habitantes, o que ocorreria se estivessem instituídas, de forma efetiva, a 
microrregionalização e a regulação da atenção. Essas questões estavam previstas quando da edição da PNHPP.

Com relação à atuação desses hospitais na região estudada, verificou-se que, após mais de 10 anos da publicação da PNHPP, dos 14 HPP existentes e que atendiam aos critérios de adesão, oito aderiram à essa política.

Ao analisar os parâmetros recomendados para HPP quanto ao número de internações, considerando a necessidade de 0,05 internações/habitante/ano (ou seja, estima-se que $5 \%$ da população deveria ser internada nos HPP/ano), conclui-se que deveriam ter sido realizadas 7028 internações nos 14 HPP da região para atender essa estimativa. Ainda segundo estes parâmetros assistenciais estabelecidos para os HPP, essas internações deveriam se distribuir em $20 \%$ cirúrgicas, 30\% clínicas, 30\% obstétricas e $20 \%$ pediátricas. Ocorre que nestes hospitais ocorreu um número bem menor de internações (3918) no ano de 2018. Em relação à distribuição de internações, os percentuais entre as clínicas também não ocorreram segundo os parâmetros estabelecidos, sendo de $0,9 \%$ para a cirúrgica, $83,3 \%$ clínica, 6,1\% para internações obstétricas e $5,2 \%$ para as pediátricas.

Quanto à distribuição das internações, chama atenção o fato de todos hospitais terem salas cirúrgicas, mas apenas três hospitais terem realizado tais internações, sendo que, em apenas um deles ocorreu mais de 20 internações deste tipo (em média) no ano de 2018, permitindo inferir que apenas neste ocorreram cirurgias rotineiramente. No estudo de Sales e Costa ${ }^{15}$, realizado em um HPP no Estado da Bahia, também identificou uma baixa taxa de internação hospitalar, sendo a média de 12 internamentos por mês. Já no estudo de Silva ${ }^{16}$ realizado com hospitais de diferentes portes, verificou-se que a menor taxa de internação foi nos HPP.

Como já exposto, a maioria realiza apenas internações na urgência, o que leva a deduzir que as internações praticamente se dão após atendimento em seus serviços de Pronto Atendimento/ Pronto Socorro. E, embora o número de internação em caráter de urgência tenha sido alto, apenas quatro desses hospitais declararam serviço especializado de urgência e emergência no CNES. Nesse sentido, cabe questionar: que papel está sendo desempenhado por esses hospitais na Rede de Urgência e Emergência (RUE)?

O papel do HPP depende da região onde ele se situa, das necessidades e do perfil epidemiológico da população local. São as características da região que devem determinar isso ${ }^{5}$. Dessa forma, parece não estar sendo cumprido papel específico por esses hospitais na RUE, nem mesmo após a contratualização, visto que não atendem os requisitos preconizados dentre os vários eixos de inserção dos hospitais nas RAS, mas somente os interesses da gestão local ${ }^{6,7}$.

Ainda sobre a inserção dos HPP nas RAS, analisando 0 desempenho destes na Rede Materno-infantil, denominada no Estado de Rede Mãe Paranaense, verificou-se que os hospitais de Bela Vista do Paraíso, Florestópolis, Jaguapitã e Porecatu eram, no ano de 2018, referência no parto de risco habitual para esta Rede. Ao analisar os resultados constatou-se que apenas o hospital de Bela Vista do Paraíso tinha profissionais médicos ginecologista/obstetra e pediatra cadastrados no CNES. O Hospital de Florestópolis tinha apenas pediatra e os dois últimos, apesar de estarem integrados nesta Rede, inclusive como referência para outros municípios, não contavam nem com pediatra nem com ginecologista/obstetra castrado no CNES, o que leva a inferir que estes procedimentos foram realizados por médicos generalistas. Em relação ao atendimento obstétrico, apenas Bela Vista do Paraíso realizou número significativo de internações obstétricas no ano de 2018 (164), o que pressupõe a participação efetiva apenas deste hospital na Rede Mãe Paranaense.

De acordo com Silva ${ }^{16}$ os HPP não se caracterizam como um ponto de atenção em rede, ou seja, não possuem um papel claro de sua missão na rede em que estão inseridas.

O valor médio da internação dos hospitais em estudo ( $R \$ 428,70$ ), encontra-se acima do encontrado por Ugá e López ${ }^{4}$, que foi de $R \$ 342,00$. Cabe destacar que nesse período não houve reajuste significativo nos valores das internações pela tabela do SUS.

O tempo de internação encontrado corroborou os achados de Ramos et al. ${ }^{1}$ onde os HPP que prestavam assistência ao SUS no estado de São Paulo tiveram internação com duração média de 3,7 dias. 
Enfim, destaca-se a extrema ociosidade da capacidade instalada, centros cirúrgicos praticamente inativos, leitos hospitalares com baixíssima ocupação. Ao contrário, nos maiores centros urbanos as filas de cirurgias são enormes, os leitos de hospitais secundários e terciários quase sempre totalmente ocupados, muitas vezes com internações que poderiam ser resolvidas em HPP, encarecendo os custos finais e reduzindo os recursos direcionados para a Atenção Básica ${ }^{17}$.

Em um cenário nacional marcado por diversas crises: econômica - que se manifesta pelo subfinanciamento público e ao mesmo tempo pelo uso inadequado dos recursos, incluindo a corrupção ${ }^{18}$; política - pela dificuldade de articulação de múltiplos atores de três entes federados, pela descontinuidade de políticas públicas a cada troca de governo; e sanitária- onde impera a tripla carga de doenças (doenças agudas e crônicas e traumas), oferta insuficiente de serviços e pouca articulação entre os mesmos ${ }^{5,17}$; esses hospitais públicos deveriam desempenhar um papel estratégico. Porém, ao invés disso, a atuação dos HPP potencializa ainda mais esse contexto de crises.

Diante disso, Silva ${ }^{16}$ menciona a redefinição do perfil dos HPP e a adoção de modelo da gerência/gestão com base em governança locorregional como um possível caminho para o aumento da eficiência e a redução de custos destas unidades. Costa e Sales ${ }^{15}$, reforçam a necessidade de estabelecer medidas que promovam a integração e inserção do HPP com outras RAS, bem como recomenda a articulação com outros entes federativos a fim de apoiar tais estruturas quanto à sua qualificação.

É de fundamental importância também que os HPP estejam organizados de forma coordenada com o sistema de saúde, evitando superposições de atividades e ineficiência, o que acarretaria numa maior presença destes no cenário de discussão e execução das políticas de saúde, rumo aos princípios e diretrizes do SUS.

\section{CONSIDERAÇÕES FINAIS}

Passados 15 anos da edição da PNHPP, verifica-se que seus objetivos não foram plenamente alcançados, ao menos na região estudada. $O$ desempenho dos HPP, considerando os indicadores analisados apresenta-se abaixo do preconizado, e a inserção destes nas RAS ainda é incipiente.

Conclui-se assim, que a forma de pactuação/ contratualização isolada de cada instituição hospitalar com a SESA não surtiu os efeitos esperados. Para superar essa situação, sugere-se que a pactuação/contratualização ocorra de forma regionaliza$\mathrm{da}$, ou seja, que os gestores municipais, detentores da maioria dos hospitais, criem um sistema de gestão unificado, onde hospitais estrategicamente localizados em microrregiões fossem melhor equipados, inclusive com transferência de equipamentos de outros hospitais, e que estes últimos passassem a atuar como Unidades de Pronto Atendimento.

Além de melhor adequação física e de equipamentos desses hospitais microrregionais, há a necessidade de investir na qualificação das equipes médicas, seja por meio de ações de educação continuada, seja por meio de ações de educação permanente em saúde, em especial de áreas cirúrgicas para atenderem pacientes, não apenas de uma microrregião, mas de uma região inteira, englobando uma ou mais especialidades cirúrgicas, descomprimindo assim a demanda dos grandes centros.

Para operacionalizar a gestão dos hospitais, os municípios poderiam viabilizar a criação de um consórcio de saúde específico, nos mesmos moldes dos existentes ${ }^{19}$. Nesse sentido, há a possibilidade também da criação de uma empresa pública ou algum outro tipo de ente jurídico público ou privado sem fins lucrativos, que administrasse os hospitais municipais através de instrumentos legais adequados. Essa proposta é interessante do ponto de vista de melhoria da gestão, uma vez que poderiam ser implantados serviços centralizados de compras, recursos humanos, manutenção, treinamentos, entre outros, com ganhos em escala. Da mesma forma, podem ser implantados serviços de custos, avaliações, controles e auditorias para potencializar o acompanhamento do desempenho de cada serviço.

As propostas de mudança de gestão deverão ser trabalhadas regionalmente, em cada hospital e com a comunidade, em uma finalidade conjunta de transformação dos hospitais em pronto atendimentos, o que não significará perda do hospital local, mas ao racionalizar e otimizar recursos haveria melhoria na qualidade da atenção e maior resolutividade dos serviços. 


\section{REFERÊNCIAS}

1. Ramos MC de A et al. Avaliação de desempenho de hospitais que prestam atendimento pelo sistema público de saúde, Brasil. Rev Saúde Públ 2015;49:43.

2. Negri Filho A, Barbosa Z. O papel dos hospitais nas redes de atenção à saúde. Elementos para pensar uma Agenda Estratégica para o SUS. Consensus: Revista do Conselho Nacional de Secretários de Saúde 2014;4(11): 42-49.

3. Proadess. Monitoramento da assistência hospitalar no Brasil (2009-2017). Boletim Informativo do Proadess, no 4, fev./2019. Disponível em: <https:// www.proadess.icict.fiocruz.br/Boletim_4_PROADESS_ Monitoramento \%20da\%20assistencia\%20hospitalar_ errata_1403.pdf>. Acesso em: 10 jun 2020.

4. Uga MAD, Lopez EM. Os hospitais de pequeno porte e sua inserção no SUS. Cien Saude Colet 2007;12(4):915-928.

5. Carpanez, L.R; Malik, A.M. O efeito da municipalização no sistema hospitalar brasileiro: Os hospitais de pequeno porte. Ciência \& Saúde Coletiva (prelo), 2019.

6. Rosa T. O papel do hospital na Rede de Atenção à Saúde. Consensus: Revista do Conselho Nacional de Secretários de Saúde 2014;4(11):13-22.

7. Souza FEA de, Nunes E de FP de A, Carvalho BG, Mendonça $F$ de $F$, Lazarini FM. Atuação dos hospitais de pequeno porte de pequenos municípios nas redes de atenção à saúde. Saude soc. 2019; 28(3):143-156.

8. La forgia GM, Couttolenc BF. Desempenho hospitalar no Brasil: em busca da excelência. São Paulo: Singular; 2009.

9. Brasil. Portaria no 1044/GM, de $1^{\circ}$ de junho de 2004. Disponível em: <http://bvsms.saude.gov.br/bvs/publicacoes/portaria_1044_ac.htm>. Acesso em: 14 Jan. 2018
10. Bittar O, Mendes J. Hospital, fetiche e decepção. Valor Econômico. Disponível em: http://portal.saude.sp.gov. $\mathrm{br} /$ resources/ses/perfil/profissional-da-saude/destaques//hospital_fetiche_e_decepcao.pdf. Acesso em 10 janeiro de $201 \overline{8}$.

11. Major MJ, Magalhães A. Reestruturação do serviço nacional de saúde em Portugal: balanço da empresarialização dos hospitais públicos portugueses R.Adm. 2014;49(3)476-490.

12. Bernardo M, Valls J, Casadesus M. Strategic alliances: an analysis of Catalan hospitals. Rev Panam Salud Publica. 2012;31(1):40-7.

13. Brasil. Portaria no 1.101, de 12 de junho de 2002. Disponível em: http://bvsms.saude.gov.br/bvs/saudelegis/gm/2002/prt1101_12_06_2002.html. Acesso em 5 Janeiro de 2018.

14. Bourdieu P. O poder simbólico. Rio de Janeiro: Ed. Bertrand Brasil; 1989.

15. Costa MO, Sales RV. Avaliação de Política Pública para Hospitais de Pequeno Porte: um referencial para a efetividade. Revista de Políticas Públicas, 2019;23(2):771-785.

16. Silva JPT. Análise da eficiência de hospitais regionais em um estado do Nordeste. Saúde debate.2019;43(120): 84-97.

17. Correa LRC. Os hospitais de pequeno porte do sistema único de saúde brasileiro e a segurança do paciente. [Dissertação de Mestrado em Administração de Empresas] São Paulo: Fundação Getúlio Vargas; 2009.

18. Chisholm D, Evans DB. Improving health system efficiency as a means of moving towards universal coverage. World health report: Background Paper n.28. Suiça: OMS; 2010.

19. Neves LA, Ribeiro JM. Consórcios de saúde: estudo de caso exitoso. Cad Saúde Pública 2006;22(10): 2207-2217. 